\title{
Scattering properties of a parity-time-antisymmetric non-Hermitian system
}

\author{
L. Jin* \\ School of Physics, Nankai University, Tianjin 300071, China
}

(Received 17 December 2017; published 13 August 2018)

\begin{abstract}
We investigate the scattering properties of a parity-time-antisymmetric non-Hermitian system. The paritytime-antisymmetric scattering center possesses imaginary nearest-neighbor hoppings and real on-site potentials; it has been experimentally realized through dissipative coupling and frequency detuning between atomic spin waves. We find that such parity-time-antisymmetric system displays three salient features: First, the reflection and transmission are both reciprocal. Second, the reflection and transmission probabilities satisfy $R \pm T=1$, which depends on the parity of the scattering center size. Third, the scattering matrix satisfies $\left(S \sigma_{\mathrm{z}}\right)\left(S \sigma_{\mathrm{z}}\right)^{*}=I$ for a scattering center with an even site; for a scattering center with an odd site, the dynamics exhibits Hermitian scattering behavior, possessing unitary scattering matrix $S S^{\dagger}=I$.
\end{abstract}

DOI: 10.1103/PhysRevA.98.022117

\section{INTRODUCTION}

The concept of parity-time $(\mathcal{P} \mathcal{T})$ symmetry has been studied for more than two decades; researchers are interested in the peculiar effects caused by $\mathcal{P} \mathcal{T}$ symmetry in non-Hermitian systems [1-12]. The $\mathcal{P} \mathcal{T}$-symmetry breaking was demonstrated in coupled passive optical waveguides with different losses [13]. By applying a pump beam to one waveguide, an active $\mathcal{P} \mathcal{T}$-symmetric system was realized, and the light power oscillation in the exact $\mathcal{P} \mathcal{T}$-symmetric phase was observed [14]. In $2014, \mathcal{P} \mathcal{T}$ symmetry was first experimentally demonstrated in coupled optical microcavities [15]. The gain is induced by lasing from the doped $\mathrm{Er}^{3+}$ ions under pumping. A single-mode operation after selectively breaking the $\mathcal{P} \mathcal{T}$ symmetry enhances the mode gain [16,17]. The modes are chiral at the exceptional point and lasing direction is controllable [18]. Recently, the enhancement of sensing has been demonstrated near the exceptional points of $\mathcal{P} \mathcal{T}$-symmetric systems $[19,20]$.

Symmetry in physical systems usually leads to symmetric physical properties. $\mathcal{P} \mathcal{T}$ symmetry induces reciprocal scattering [21-25]. Reflection $\mathcal{P} \mathcal{T}$ symmetry protects the reciprocal transmission; axial $\mathcal{P} \mathcal{T}$ symmetry protects the reciprocal reflection $[26,27]$. In the presence of non-Hermiticity, the scattering is not unitary in a general situation, leading to nonreciprocal reflection (transmission) for a reciprocal transmission (reflection). $\mathcal{P} \mathcal{T}$ symmetry and non-Hermiticity are the key points of the nonreciprocal scattering behavior exhibited in a $\mathcal{P} \mathcal{T}$-symmetric system. Many intriguing phenomena have been observed such as coherent perfect absorption [28-31], unidirectional invisibility, and reflectionless [32-34] and spectral singularity [35]. Until now, the scattering properties of

\footnotetext{
*jinliang@nankai.edu.cn

Published by the American Physical Society under the terms of the Creative Commons Attribution 4.0 International license. Further distribution of this work must maintain attribution to the author(s) and the published article's title, journal citation, and DOI.
}

a system with $\mathcal{P} \mathcal{T}$ symmetry have been explicit, however, $\mathcal{P} \mathcal{T}$ antisymmetry as a counterpart of $\mathcal{P} \mathcal{T}$ symmetry has rarely been investigated [36-41]. Recently, the imaginary coupling was experimentally realized through dissipative coupling between atomic vapors. The system is non-Hermitian and satisfies $\mathcal{P} \mathcal{T}$ antisymmetry. The phase-transition threshold and reflectionless light propagation have been observed in high resolution [37].

In this paper, inspired by the experimentally realized $\mathcal{P} \mathcal{T}$ antisymmetric system, we study the scattering properties of a $\mathcal{P} \mathcal{T}$-antisymmetric non-Hermitian system, which has imaginary couplings and real on-site potentials. We demonstrate that the reflection and transmission are both reciprocal. Besides, the difference or summation between the reflection and transmission probabilities is unity, and this relation depends on the parity of the scattering center. The scattering matrix satisfies $\left(S \sigma_{\mathrm{z}}\right)\left(S \sigma_{\mathrm{z}}\right)^{*}=I$ or $S S^{\dagger}=I$ for the scattering center with even or odd site, respectively. In the latter case, the $\mathcal{P} \mathcal{T}$-antisymmetric non-Hermitian system exhibits Hermitian scattering behavior.

The remainder of the paper is as follows. In Sec. II, the system is modeled. In Sec. III, the scattering properties of a $\mathcal{P} \mathcal{T}$-antisymmetric non-Hermitian system are demonstrated. In Sec. IV, two concrete examples are presented as an illustration. The results are summarized and discussed in Sec. V.

\section{MODEL}

Recently, a $\mathcal{P} \mathcal{T}$-antisymmetric non-Hermitian system has been realized in atomic vapors [37]. A novel coupling mechanism leads to a dissipative coupling between two atomic spin waves. In its Hamiltonian, the dissipative coupling is the imaginary coupling and the detuning between two atomic spin waves is the on-site potential. In this work, we study the scattering properties of a $\mathcal{P} \mathcal{T}$-antisymmetric scattering center, which is a tight-binding chain with imaginary couplings and real on-site potentials (Fig. 1). The Hamiltonian of the 


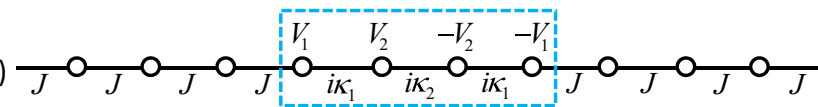

$H_{\mathrm{L}}$ $H_{\mathrm{c}}$

$H_{\mathrm{R}}$

(b)

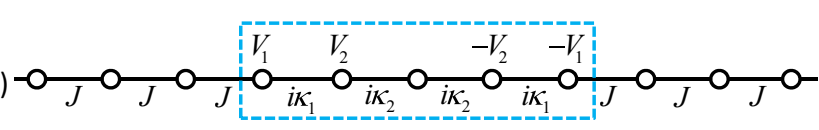

FIG. 1. Schematic illustration of a $\mathcal{P} \mathcal{T}$-antisymmetric scattering system. The site number of the scattering center is (a) even and (b) odd.

scattering center reads

$$
H_{\mathrm{c}}=\sum_{j=1}^{N} i \kappa_{j}\left(|j\rangle_{\mathrm{cc}}\langle j+1|+| j+1\rangle_{\mathrm{cc}}\langle j|\right)+V_{j}|j\rangle_{\mathrm{cc}}\langle j|,
$$

where the couplings satisfy $\kappa_{j}=\kappa_{N+1-j}$ and the on-site potentials satisfy $V_{j}=-V_{N+1-j} \cdot|j\rangle_{\mathrm{c}}$ is the basis of the scattering center site $j$. The parity operator $\mathcal{P}$ is defined as the space reflection $\mathcal{P} j \mathcal{P}^{-1}=N+1-j ; \mathcal{T}$ is defined as the time-reversal operator $\mathcal{T} i \mathcal{T}^{-1}=-i$. Under these definitions, the scattering center $H_{\mathrm{c}}$ possesses $\mathcal{P} \mathcal{T}$ antisymmetry, which satisfies $(\mathcal{P} \mathcal{T}) H_{\mathrm{c}}(\mathcal{P} \mathcal{T})^{-1}=-H_{\mathrm{c}}$. Notably, it is interesting that the $\mathcal{P} \mathcal{T}$-antisymmetric Hamiltonian $H_{\mathrm{c}}$ satisfies $(\mathcal{P} \mathcal{T})\left( \pm i H_{\mathrm{c}}\right)(\mathcal{P} \mathcal{T})^{-1}= \pm i H_{\mathrm{c}}$, which indicates that Hamiltonians $\pm i H_{\mathrm{c}}$ are $\mathcal{P} \mathcal{T}$ symmetric.

The input and output leads are connected to the scattering center. The Hamiltonian of the system is in the form of $H=$ $H_{-}+H_{+}+H_{\mathrm{c}}+H_{\text {in }}$, where

$$
H_{ \pm}=J \sum_{j= \pm 1}^{ \pm \infty}\left(|j \pm 1\rangle_{11}\langle j|+\text { H.c. }\right)
$$

are the input and output leads with uniform coupling strength $J .|j\rangle_{1}$ is the basis of the leads site $j$.

$$
H_{\text {in }}=g|-1\rangle_{\text {lc }}\langle 1|+g| N\rangle_{\mathrm{cl}}\langle+1|+\text { H.c. }
$$

is the connection Hamiltonian. $|1\rangle_{\mathrm{c}}$ and $|N\rangle_{\mathrm{c}}$ are the sites of the scattering center $H_{\mathrm{c}}$ that connected to the input and output leads $H_{-}$and $H_{+}$, respectively.

\section{SCATTERING FORMALISM}

In this section, we investigate the scattering properties of a $\mathcal{P} \mathcal{T}$-antisymmetric non-Hermitian scattering center, in which typical scattering behaviors are revealed. In the following, we discuss the scattering properties of the $\mathcal{P} \mathcal{T}$-antisymmetric scattering center through investigation of the reflection and transmission of the left and the right inputs. The wave function for the left input is denoted as $\psi_{\mathrm{L}}^{k}(j)$ and for the right input is denoted as $\psi_{\mathrm{R}}^{k}(j)$ for site $|j\rangle_{\mathrm{c}}$, where $k$ is the wave vector. The wave functions are in the form of

$$
\begin{aligned}
& \psi_{\mathrm{L}}^{k}(j)= \begin{cases}e^{i k j}+r_{\mathrm{L}} e^{-i k j}, & j<0 \\
t_{\mathrm{L}} e^{i k j}, & j>0,\end{cases} \\
& \psi_{\mathrm{R}}^{k}(j)= \begin{cases}t_{\mathrm{R}} e^{-i k j}, & j<0 \\
e^{-i k j}+r_{\mathrm{R}} e^{i k j}, & j>0,\end{cases}
\end{aligned}
$$

where $r_{\mathrm{L}}\left(t_{\mathrm{L}}\right)$ and $r_{\mathrm{R}}\left(t_{\mathrm{R}}\right)$ are the reflection (transmission) coefficients for the left and right inputs, respectively.

\section{A. Identical transmission of transpose invariant}

The scattering center satisfies transpose invariant, i.e., $H_{\mathrm{c}}=H_{\mathrm{c}}^{T}$. This leads to identical left and right transmission coefficients, i.e., $t_{\mathrm{L}}=t_{\mathrm{R}}$. For the left input, the Schrödinger equations for the scattering center are in the form of

$$
\left(H_{\mathrm{c}}-E I_{N \times N}\right) \Psi_{\mathrm{c}, \mathrm{L}}=\Phi_{\mathrm{c}, \mathrm{L}},
$$

where $E=2 J \cos k$ is the dispersion relation obtained from the Schrödinger equations for the leads; $I_{N \times N}$ is the $(N \times N)$ dimension identical matrix. $\Psi_{\mathrm{c}, \mathrm{L}}$ and $\Phi_{\mathrm{c}, \mathrm{L}}$ are $N$-dimension column vectors and their elements are $\Psi_{\mathrm{c}, \mathrm{L}}(j)=\psi_{\mathrm{c}}^{k}(j)$ for $j \in[1, N] . \psi_{\mathrm{c}}^{k}(j)$ represents the wave function of site $j$ in the scattering center $H_{\mathrm{c}} . \Phi_{\mathrm{c}, \mathrm{L}}(1)=-g \psi_{\mathrm{L}}^{k}(-1), \Phi_{\mathrm{c}, \mathrm{L}}(N)=$ $-g \psi_{\mathrm{L}}^{k}(+1)$, and $\Phi_{\mathrm{c}, \mathrm{L}}(j)=0$ for $j \in[2, N-1]$. The wave functions at site \pm 1 are $\psi_{\mathrm{L}}^{k}(-1)=e^{-i k}+r_{\mathrm{L}} e^{i k}$ and $\psi_{\mathrm{L}}^{k}(+1)=$ $t_{\mathrm{L}} e^{i k}$. From Eq. (6), we have

$$
\begin{gathered}
\Psi_{\mathrm{c}, \mathrm{L}}(1)=\Delta_{11}^{-1} \Phi_{\mathrm{c}, \mathrm{L}}(1)+\Delta_{1 N}^{-1} \Phi_{\mathrm{c}, \mathrm{L}}(N), \\
\Psi_{\mathrm{c}, \mathrm{L}}(N)=\Delta_{N 1}^{-1} \Phi_{\mathrm{c}, \mathrm{L}}(1)+\Delta_{N N}^{-1} \Phi_{\mathrm{c}, \mathrm{L}}(N),
\end{gathered}
$$

where $\Delta=H_{\mathrm{c}}-E I_{N \times N}$, and $\Delta_{m n}^{-1}$ represents the element of matrix $\Delta^{-1}$ on the $m$ th row and $n$th column. Then, we have

$$
\begin{gathered}
-\frac{\psi_{\mathrm{c}}^{k}(1)}{g}=\Delta_{11}^{-1}\left(e^{-i k}+r_{\mathrm{L}} e^{i k}\right)+\Delta_{1 N}^{-1} t_{\mathrm{L}} e^{i k}, \\
-\frac{\psi_{\mathrm{c}}^{k}(N)}{g}=\Delta_{N 1}^{-1}\left(e^{-i k}+r_{\mathrm{L}} e^{i k}\right)+\Delta_{N N}^{-1} t_{\mathrm{L}} e^{i k} .
\end{gathered}
$$

The Schrödinger equations for the lead sites $|-1\rangle_{1}$ and $|+1\rangle_{1}$ yield

$$
\begin{gathered}
J \psi_{\mathrm{L}}^{k}(-2)+g \psi_{\mathrm{c}}^{k}(1)=E \psi_{\mathrm{L}}^{k}(-1), \\
J \psi_{\mathrm{L}}^{k}(+2)+g \psi_{\mathrm{c}}^{k}(N)=E \psi_{\mathrm{L}}^{k}(1),
\end{gathered}
$$

and the wave functions at sites \pm 2 are $\psi_{\mathrm{L}}^{k}(-2)=e^{-2 i k}+$ $r_{\mathrm{L}} e^{2 i k}$ and $\psi_{\mathrm{L}}^{k}(+2)=t_{\mathrm{L}} e^{2 i k}$. Then, we have

$$
\psi_{\mathrm{c}}^{k}(1)=\frac{J}{g}\left(1+r_{\mathrm{L}}\right), \quad \psi_{\mathrm{c}}^{k}(N)=\frac{J}{g} t_{\mathrm{L}} .
$$

The two kinds of expressions for $\psi_{\mathrm{c}}^{k}(1)$ and $\psi_{\mathrm{c}}^{k}(N)$ are equivalent, and therefore,

$$
\begin{gathered}
-\frac{J\left(1+r_{\mathrm{L}}\right)}{g^{2}}=\Delta_{11}^{-1}\left(e^{-i k}+r_{\mathrm{L}} e^{i k}\right)+\Delta_{1 N}^{-1} t_{\mathrm{L}} e^{i k} \\
-\frac{J}{g^{2}} t_{\mathrm{L}}=\Delta_{N 1}^{-1}\left(e^{-i k}+r_{\mathrm{L}} e^{i k}\right)+\Delta_{N N}^{-1} t_{\mathrm{L}} e^{i k}
\end{gathered}
$$

and the transmission for the left input is

$$
t_{\mathrm{L}}=\frac{2 i\left(J / g^{2}\right) \Delta_{N 1}^{-1} \sin k}{\left[\frac{J}{g^{2}}+\Delta_{N N}^{-1} e^{i k}\right]\left[\frac{J}{g^{2}}+\Delta_{11}^{-1} e^{i k}\right]-\Delta_{N 1}^{-1} \Delta_{1 N}^{-1} e^{2 i k}} .
$$


For the right input, the Schrödinger equations for the scattering center are in the form of

$$
\Delta \Psi_{\mathrm{c}, \mathrm{R}}=\Phi_{\mathrm{c}, \mathrm{R}},
$$

where $\Psi_{\mathrm{c}, \mathrm{R}}$ and $\Phi_{\mathrm{c}, \mathrm{R}}$ are $N$-dimension column vectors, and their elements are $\Psi_{\mathrm{c}, \mathrm{R}}(j)=\psi_{\mathrm{c}}^{k}(j)$ for $j \in[1, N] ; \Phi_{\mathrm{c}, \mathrm{R}}(1)=$ $-g \psi_{\mathrm{R}}^{k}(-1), \Phi_{\mathrm{c}, \mathrm{R}}(N)=-g \psi_{\mathrm{R}}^{k}(+1)$, and $\Phi_{\mathrm{c}, \mathrm{R}}(j)=0$ for $j \in$ $[2, N-1]$. The wave functions at sites \pm 1 are $\psi_{\mathrm{R}}^{k}(-1)=t_{\mathrm{R}} e^{i k}$ and $\psi_{\mathrm{R}}^{k}(+1)=e^{-i k}+r_{\mathrm{R}} e^{i k}$. From Eq. (6), we have

$$
\begin{gathered}
\Psi_{\mathrm{c}, \mathrm{R}}(1)=\Delta_{11}^{-1} \Phi_{\mathrm{c}, \mathrm{R}}(1)+\Delta_{1 N}^{-1} \Phi_{\mathrm{c}, \mathrm{R}}(N), \\
\Psi_{\mathrm{c}, \mathrm{R}}(N)=\Delta_{N 1}^{-1} \Phi_{\mathrm{c}, \mathrm{R}}(1)+\Delta_{N N}^{-1} \Phi_{\mathrm{c}, \mathrm{R}}(N),
\end{gathered}
$$

that is,

$$
\begin{gathered}
-\frac{\psi_{\mathrm{c}}^{k}(1)}{g}=\Delta_{11}^{-1} t_{\mathrm{L}} e^{i k}+\Delta_{1 N}^{-1}\left(e^{-i k}+r_{\mathrm{R}} e^{i k}\right), \\
-\frac{\psi_{\mathrm{c}}^{k}(N)}{g}=\Delta_{N 1}^{-1} t_{\mathrm{L}} e^{i k}+\Delta_{N N}^{-1}\left(e^{-i k}+r_{\mathrm{R}} e^{i k}\right) .
\end{gathered}
$$

The Schrödinger equations for the lead sites $|-1\rangle_{1}$ and $|+1\rangle_{1}$ yield

$$
\begin{gathered}
J \psi_{\mathrm{R}}^{k}(-2)+g \psi_{\mathrm{c}}^{k}(1)=E \psi_{\mathrm{R}}^{k}(-1), \\
J \psi_{\mathrm{R}}^{k}(+2)+g \psi_{\mathrm{c}}^{k}(N)=E \psi_{\mathrm{R}}^{k}(+1),
\end{gathered}
$$

and the wave functions at sites \pm 2 are $\psi_{\mathrm{R}}^{k}(-2)=t_{\mathrm{R}} e^{2 i k}$ and $\psi_{\mathrm{R}}^{k}(+2)=e^{-2 i k}+r_{\mathrm{R}} e^{2 i k}$. Then, we have

$$
\psi_{\mathrm{c}}^{k}(1)=\frac{J}{g} t_{\mathrm{R}}, \quad \psi_{\mathrm{c}}^{k}(N)=\frac{J}{g}\left(1+r_{\mathrm{R}}\right),
$$

and therefore,

$$
\begin{gathered}
-\frac{J}{g^{2}} t_{\mathrm{R}}=\Delta_{11}^{-1} t_{\mathrm{L}} e^{i k}+\Delta_{1 N}^{-1}\left(e^{-i k}+r_{\mathrm{R}} e^{i k}\right), \\
-\frac{J\left(1+r_{\mathrm{R}}\right)}{g^{2}}=\Delta_{N 1}^{-1} t_{\mathrm{L}} e^{i k}+\Delta_{N N}^{-1}\left(e^{-i k}+r_{\mathrm{R}} e^{i k}\right),
\end{gathered}
$$

and the transmission for the right input is

$$
t_{\mathrm{R}}=\frac{2 i\left(J / g^{2}\right)\left(\Delta^{-1}\right)_{1 N} \sin k}{\left[\frac{J}{g^{2}}+\Delta_{11}^{-1} e^{i k}\right]\left[\frac{J}{g^{2}}+\Delta_{N N}^{-1} e^{i k}\right]-\Delta_{1 N}^{-1} \Delta_{N 1}^{-1} e^{2 i k}} .
$$

Because $H_{\mathrm{c}}=H_{\mathrm{c}}^{T}$, we have $\Delta=\Delta^{T}$. Notice that $\left(\Delta^{T}\right)^{-1}=\left(\Delta^{-1}\right)^{T}$; then we obtain $\Delta^{-1}=\left(\Delta^{T}\right)^{-1} \Delta^{T} \Delta^{-1}=$ $\left(\Delta^{T}\right)^{-1}=\left(\Delta^{-1}\right)^{T}$. Thus, the matrix elements satisfy $\Delta_{1 N}^{-1}=$ $\Delta_{N 1}^{-1}$. Through comparing Eqs. (16) and (27), we notice that the left transmission coefficient is identical with the right transmission coefficient. Therefore, the transpose invariant of $H_{\mathrm{c}}$ yields identical transmission coefficients:

$$
t_{\mathrm{L}}=t_{\mathrm{R}}
$$

\section{B. Reciprocal reflection under $\mathcal{T}$ symmetry}

The scattering center is also invariant under a time-reversal operation. The time-reversal operator can be expressed as unitary operator $\mathcal{U}$ multiples of the complex conjugation operator $\mathcal{K}$, i.e., $\mathcal{T}=\mathcal{U} \mathcal{K}$. The element of the unitary operator
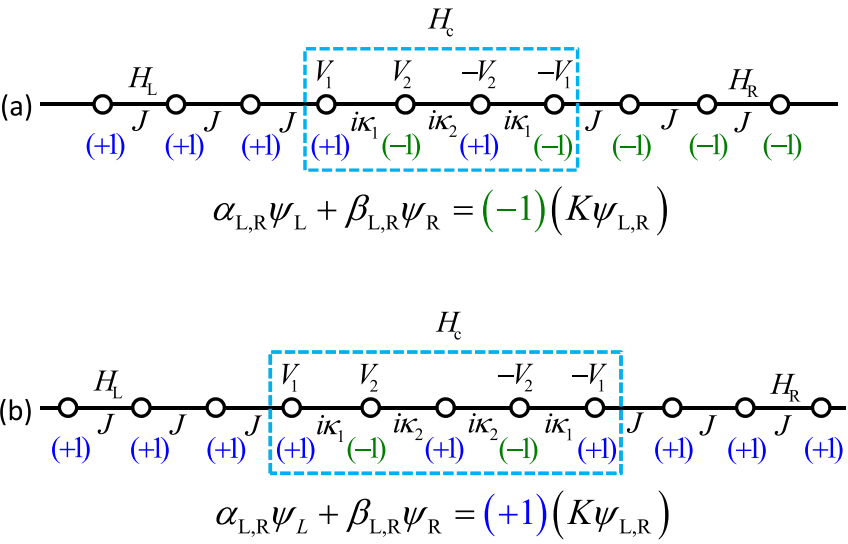

FIG. 2. The wave-function relation between the $\mathcal{P} \mathcal{T}$ antisymmetric scattering system. The site number of the center is (a) even and (b) odd. The +1 in blue and -1 in green represent the unitary transformation; the scattering system changes back to itself after a time-reversal operation.

$\mathcal{U}$ is ${ }_{c}\langle m|\mathcal{U}| n\rangle_{\mathrm{c}}=(-1)^{m-1} \delta(m-n)$, where $\delta$ is the Dirac delta function.

The unitary operator is a diagonal matrix with staggered elements +1 and -1 , which is a transformation on the scattering center basis. We schematically illustrate this basis transformation in Fig. 2 with the coefficients +1 in blue and -1 in green. +1 indicates that the basis is unchanged; -1 indicates that the basis changes from $|j\rangle_{\mathrm{c}}$ to $-|j\rangle_{\mathrm{c}}$ after the basis transformation. Figure 2 implies the Hamiltonian of the scattering center $H_{\mathrm{c}}$ is invariant after the time-reversal operation, i.e., acting on the complex conjugation $\mathcal{K}$ and the basis transformation. To make the whole system Hamiltonian $H$ invariant after the time-reversal operation, the basis on the left and right leads needs to change accordingly. The basis on the left lead is unchanged, but changes from $|j\rangle_{1}$ to $-|j\rangle_{1}$ on the right lead for the scattering center with even site. To make the whole system Hamiltonian $H$ unchanged after a time-reversal operation, the coefficients on the basis of the two leads for the scattering center with even site ( $N$ is even) are opposite [Fig. 2(a)]; the basis of the two leads for the scattering center with odd site ( $N$ is odd) is unchanged [Fig. 2(b)]. This difference indicates two distinct relations of the scattering wave functions (Fig. 2). The left input and the right input wave functions with identical wave vector $k$ can compose either the left or the right wave function after a time-reversal operation in two alternative ways for the scattering center with different parities.

We apply the complex conjugation operator $\mathcal{K}$ on the wave functions given by Eqs. (4) and (5) to get

$$
\begin{aligned}
\mathcal{K} \psi_{\mathrm{L}}^{k}(j) & = \begin{cases}e^{-i k j}+r_{\mathrm{L}}^{*} e^{i k j}, & j<0 \\
t_{\mathrm{L}}^{*} e^{-i k j}, & j>0,\end{cases} \\
\mathcal{K} \psi_{\mathrm{R}}^{k}(j) & = \begin{cases}t_{\mathrm{R}}^{*} e^{i k j}, & j<0 \\
e^{i k j}+r_{\mathrm{R}}^{*} e^{-i k j}, & j>0 .\end{cases}
\end{aligned}
$$

For the configuration shown in Fig. 2(a), we can compose $-\mathcal{K} \psi_{\mathrm{L}}^{k}(j)$ in the $j<0$ region through $\psi_{\mathrm{L}}^{k}(j)$ and $\psi_{\mathrm{R}}^{k}(j)$ of Eqs. (4) and (5) by eliminating $e^{i k j}$ in the $j>0$ region. We 
have

$$
\begin{aligned}
& t_{\mathrm{L}}^{*}\left[\psi_{\mathrm{R}}^{k}(j)-\frac{r_{\mathrm{R}}}{t_{\mathrm{L}}} \psi_{\mathrm{L}}^{k}(j)\right] \\
& \quad= \begin{cases}t_{\mathrm{L}}^{*}\left(t_{\mathrm{R}}-r_{\mathrm{R}} \frac{r_{\mathrm{L}}}{t_{\mathrm{L}}}\right) e^{-i k j}-t_{\mathrm{L}}^{*} \frac{r_{\mathrm{R}}}{t_{\mathrm{L}}} e^{i k j}, & j<0 \\
t_{\mathrm{L}}^{*} e^{-i k j}, & j>0,\end{cases}
\end{aligned}
$$

where the coefficients in the $j>0$ region for the composed wave function $t_{\mathrm{L}}^{*}\left[\psi_{\mathrm{R}}^{k}(j)-\frac{r_{\mathrm{R}}}{t_{\mathrm{L}}} \psi_{\mathrm{L}}^{k}(j)\right]$ and $\mathcal{K} \psi_{\mathrm{L}}^{k}(j)$ are the same, but they should be opposite in the $j<0$ region. Therefore, the coefficients in the $j<0$ region satisfy

$$
\begin{gathered}
t_{\mathrm{L}}^{*}\left(t_{\mathrm{R}}-r_{\mathrm{R}} \frac{r_{\mathrm{L}}}{t_{\mathrm{L}}}\right)=-1, \\
-t_{\mathrm{L}}^{*} \frac{r_{\mathrm{R}}}{t_{\mathrm{L}}}=-r_{\mathrm{L}}^{*} .
\end{gathered}
$$

Then we have the relations

$$
r_{\mathrm{L}} r_{\mathrm{L}}^{*}-t_{\mathrm{L}}^{*} t_{\mathrm{R}}=1 ; t_{\mathrm{L}}^{*} r_{\mathrm{R}}=r_{\mathrm{L}}^{*} t_{\mathrm{L}},
$$

for the scattering center site number that is even.

For the configuration shown in Fig. 2(b), the composed wave function $t_{\mathrm{L}}^{*}\left[\psi_{\mathrm{R}}^{k}(j)-\frac{r_{\mathrm{R}}}{t_{\mathrm{L}}} \psi_{\mathrm{L}}^{k}(j)\right]$ and $\mathcal{K} \psi_{\mathrm{L}}^{k}(j)$ are the same in both the left and the right leads. Then, we obtain

$$
\begin{gathered}
t_{\mathrm{L}}^{*}\left(t_{\mathrm{R}}-r_{\mathrm{R}} \frac{r_{\mathrm{L}}}{t_{\mathrm{L}}}\right)=1, \\
-t_{\mathrm{L}}^{*} \frac{r_{\mathrm{R}}}{t_{\mathrm{L}}}=r_{\mathrm{L}}^{*},
\end{gathered}
$$

and the relations

$$
r_{\mathrm{L}} r_{\mathrm{L}}^{*}+t_{\mathrm{L}}^{*} t_{\mathrm{R}}=1 ; \quad t_{\mathrm{L}}^{*} r_{\mathrm{R}}=-r_{\mathrm{L}}^{*} t_{\mathrm{L}}
$$

for the scattering center site number that is odd.

For the configuration shown in Fig. 2(a), we compose $-\mathcal{K} \psi_{\mathrm{R}}^{k}(j)$ via $\psi_{\mathrm{L}}^{k}(j)$ and $\psi_{\mathrm{R}}^{k}(j)$ of Eqs. (4) and (5) by eliminating $e^{-i k j}$ in the $j<0$ region. We have

$$
\begin{aligned}
t_{\mathrm{R}}^{*} & {\left[\psi_{\mathrm{L}}^{k}(j)-\frac{r_{\mathrm{L}}}{t_{\mathrm{R}}} \psi_{\mathrm{R}}^{k}(j)\right] } \\
& = \begin{cases}t_{\mathrm{R}}^{*} e^{i k j}, & j<0 \\
t_{\mathrm{R}}^{*}\left(t_{\mathrm{L}}-r_{\mathrm{L}} \frac{r_{\mathrm{R}}}{t_{\mathrm{R}}}\right) e^{i k j}-t_{\mathrm{R}}^{*} \frac{r_{\mathrm{L}}}{t_{\mathrm{R}}} e^{-i k j}, & j>0,\end{cases}
\end{aligned}
$$

where the coefficients in the $j<0$ region for the composed wave function $t_{\mathrm{R}}^{*}\left[\psi_{\mathrm{L}}^{k}(j)-\frac{r_{\mathrm{L}}}{t_{\mathrm{R}}} \psi_{\mathrm{R}}^{k}(j)\right]$ and $\mathcal{K} \psi_{\mathrm{R}}^{k}(j)$ are identical, but the coefficients in the $j>0$ region should be opposite. Therefore, we have the relations

$$
\begin{gathered}
t_{\mathrm{R}}^{*}\left(t_{\mathrm{L}}-r_{\mathrm{L}} \frac{r_{\mathrm{R}}}{t_{\mathrm{R}}}\right)=-1, \\
-t_{\mathrm{R}}^{*} \frac{r_{\mathrm{L}}}{t_{\mathrm{R}}}=-r_{\mathrm{R}}^{*} .
\end{gathered}
$$

Simplifying the obtained relations, we have

$$
r_{\mathrm{R}} r_{\mathrm{R}}^{*}-t_{\mathrm{R}}^{*} t_{\mathrm{L}}=1 ; \quad t_{\mathrm{R}}^{*} r_{\mathrm{L}}=r_{\mathrm{R}}^{*} t_{\mathrm{R}},
$$

for the scattering center site number that is even.

For the configuration shown in Fig. 2(b), the composed wave functions $t_{\mathrm{L}}^{*}\left[\psi_{\mathrm{R}}^{k}(j)-\frac{r_{\mathrm{R}}}{t_{\mathrm{L}}} \psi_{\mathrm{L}}^{k}(j)\right]$ and $\mathcal{K} \psi_{\mathrm{L}}^{k}(j)$ are the same in both the left and the right leads. Thus, we obtain

$$
\begin{gathered}
t_{\mathrm{R}}^{*}\left(t_{\mathrm{L}}-r_{\mathrm{L}} \frac{r_{\mathrm{R}}}{t_{\mathrm{R}}}\right)=1, \\
-t_{\mathrm{R}}^{*} \frac{r_{\mathrm{L}}}{t_{\mathrm{R}}}=r_{\mathrm{R}}^{*} .
\end{gathered}
$$

After simplification, we obtain the relations

$$
r_{\mathrm{R}} r_{\mathrm{R}}^{*}+t_{\mathrm{R}}^{*} t_{\mathrm{L}}=1 ; t_{\mathrm{R}}^{*} r_{\mathrm{L}}=-t_{\mathrm{R}} r_{\mathrm{R}}^{*},
$$

for the scattering center site number that is odd.

A direct conclusion from the relations of the scattering coefficients given by Eqs. (34) and (41) and Eqs. (37) and (44) is the reciprocal reflection, i.e., $\left|r_{\mathrm{L}}\right|=\left|r_{\mathrm{R}}\right|$ in both configurations of Fig. 1.

\section{Scattering probability and scattering matrix}

For the scattering center with even site, their reflection and transmission satisfy Eqs. (28), (34), and (41), from which we first obtain

$$
\left|r_{\mathrm{L}}\right|^{2}-\left|t_{\mathrm{L}}\right|^{2}=\left|r_{\mathrm{R}}\right|^{2}-\left|t_{\mathrm{R}}\right|^{2}=1 .
$$

And then, we obtain that the scattering matrix satisfies

$$
\left(S \sigma_{\mathrm{z}}\right)\left(S \sigma_{\mathrm{z}}\right)^{*}=\left(\begin{array}{ll}
r_{\mathrm{L}} r_{\mathrm{L}}^{*}-t_{\mathrm{R}} t_{\mathrm{R}}^{*} & t_{\mathrm{R}} r_{\mathrm{R}}^{*}-r_{\mathrm{L}} t_{\mathrm{L}}^{*} \\
t_{\mathrm{L}} r_{\mathrm{L}}^{*}-r_{\mathrm{R}} t_{\mathrm{R}}^{*} & r_{\mathrm{R}} r_{\mathrm{R}}^{*}-t_{\mathrm{L}} t_{\mathrm{L}}^{*}
\end{array}\right)=I_{2 \times 2},
$$

in the configuration shown in Fig. 2(a), where $S$ is the scattering matrix and $\sigma_{\mathrm{z}}$ is the Pauli matrix defined as

$$
S=\left(\begin{array}{cc}
r_{\mathrm{L}} & t_{\mathrm{R}} \\
t_{\mathrm{L}} & r_{\mathrm{R}}
\end{array}\right), \quad \sigma_{z}=\left(\begin{array}{cc}
1 & 0 \\
0 & -1
\end{array}\right) .
$$

For the scattering center with odd site, their reflection and transmission satisfy Eqs. (28), (37), and (44), from which we first obtain

$$
\left|r_{\mathrm{L}}\right|^{2}+\left|t_{\mathrm{L}}\right|^{2}=\left|r_{\mathrm{R}}\right|^{2}+\left|t_{\mathrm{R}}\right|^{2}=1 .
$$

And then, we obtain that the scattering matrix is unitary in the configuration shown in Fig. 2(b),

$$
S S^{\dagger}=\left(\begin{array}{ll}
r_{\mathrm{L}} r_{\mathrm{L}}^{*}+t_{\mathrm{R}} t_{\mathrm{R}}^{*} & r_{\mathrm{L}} t_{\mathrm{L}}^{*}+t_{\mathrm{R}} r_{\mathrm{R}}^{*} \\
t_{\mathrm{L}} r_{\mathrm{L}}^{*}+r_{\mathrm{R}} t_{\mathrm{R}}^{*} & t_{\mathrm{L}} t_{\mathrm{L}}^{*}+r_{\mathrm{R}} r_{\mathrm{R}}^{*}
\end{array}\right)=I_{2 \times 2}
$$

The scattering dynamics exhibited in the odd-site $\mathcal{P} \mathcal{T}$ antisymmetric scattering center is similar to the dynamics in a Hermitian scattering center. Therefore, unitary scattering not only occurs in a $\mathcal{P} \mathcal{T}$-symmetric non-Hermitian system [25], but also appears in a $\mathcal{P} \mathcal{T}$-antisymmetric non-Hermitian system.

\section{ILLUSTRATIVE EXAMPLES}

We consider concrete models to demonstrate our results. The two leads are $H_{ \pm}=-\sum_{j= \pm 1}^{ \pm \infty}|j \pm 1\rangle_{11}\langle j|+$ H.c.; the connection Hamiltonian is $H_{\text {in }}=-|-1\rangle_{\mathrm{lc}}\langle 1|-| 2\rangle_{\mathrm{cl}}\langle+1|+$ H.c.; and the Hamiltonian of the two-site scattering center is

$$
H_{\mathrm{c}}^{2 \mathrm{~d}}=\left(\begin{array}{cc}
V & -i \\
-i & -V
\end{array}\right) \text {. }
$$

The scattering center satisfies $(\mathcal{P} \mathcal{T}) H_{\mathrm{c}}^{2 \mathrm{~d}}(\mathcal{P} \mathcal{T})^{-1}=-H_{\mathrm{c}}^{2 \mathrm{~d}}$. The Schrödinger equations for the scattering center are

$$
\begin{aligned}
& -\psi_{\mathrm{L}(\mathrm{R})}^{k}(-1)-i \psi_{\mathrm{c}}^{k}(2)=(E-V) \psi_{\mathrm{c}}^{k}(1), \\
& -\psi_{\mathrm{L}(\mathrm{R})}^{k}(+1)-i \psi_{\mathrm{c}}^{k}(1)=(E+V) \psi_{\mathrm{c}}^{k}(2),
\end{aligned}
$$


and the dispersion is $E=-2 \cos k$. For the left input, we set the wave functions as $\psi_{\mathrm{L}}^{k}(-1)=e^{-i k}+r_{\mathrm{L}} e^{i k}, \psi_{\mathrm{c}}^{k}(1)=$ $1+r_{\mathrm{L}}, \psi_{\mathrm{c}}^{k}(2)=t_{\mathrm{L}}$, and $\psi_{\mathrm{L}}^{k}(+1)=t_{\mathrm{L}} e^{i k}$. For the right input, we set the wave functions as $\psi_{\mathrm{R}}^{k}(-1)=t_{\mathrm{R}} e^{i k}, \psi_{\mathrm{c}}^{k}(1)=t_{\mathrm{R}}$, $\psi_{\mathrm{c}}^{k}(2)=1+r_{\mathrm{R}}$, and $\psi_{\mathrm{R}}^{k}(+1)=e^{-i k}+r_{\mathrm{R}} e^{i k}$. Substituting the wave functions into the Schrödinger equations, we obtain the reflection and transmission, which read

$$
\begin{gathered}
t_{\mathrm{L}}=t_{\mathrm{R}}=\frac{2 \sin k}{2 \cos k e^{-i k}-V^{2}}, \\
r_{\mathrm{L}}=\frac{V^{2}-2+2 i V \sin k}{2 \cos k e^{-i k}-V^{2}}, \\
r_{\mathrm{R}}=\frac{V^{2}-2-2 i V \sin k}{2 \cos k e^{-i k}-V^{2}} .
\end{gathered}
$$

Notably, $t_{\mathrm{L}}=t_{\mathrm{R}},\left|r_{\mathrm{L}}\right|=\left|r_{\mathrm{R}}\right|$, and $\left|r_{\mathrm{L}(\mathrm{R})}\right|^{2}-\left|t_{\mathrm{L}(\mathrm{R})}\right|^{2}=1$. The scattering matrix satisfies $\left(S \sigma_{z}\right)\left(S \sigma_{z}\right)^{*}=I$.

For a three-site $\mathcal{P} \mathcal{T}$-antisymmetric scattering center,

$$
H_{\mathrm{c}}^{3 \mathrm{~d}}=\left(\begin{array}{ccc}
V & -i & 0 \\
-i & 0 & -i \\
0 & -i & -V
\end{array}\right),
$$

we notice that $(\mathcal{P} \mathcal{T}) H_{\mathrm{c}}^{3 \mathrm{~d}}(\mathcal{P} \mathcal{T})^{-1}=-H_{\mathrm{c}}^{3 \mathrm{~d}}$. The connection Hamiltonian is $H_{\mathrm{in}}=-|-1\rangle_{\mathrm{lc}}\langle 1|-| 3\rangle_{\mathrm{cl}}\langle+1|+$ H.c., and the Schrödinger equations are

$$
\begin{gathered}
-\psi_{\mathrm{L}(\mathrm{R})}^{k}(-1)-i \psi_{\mathrm{c}}^{k}(2)=(E-V) \psi_{\mathrm{c}}^{k}(1), \\
-i \psi_{\mathrm{c}}^{k}(1)-i \psi_{\mathrm{c}}^{k}(3)=E \psi_{\mathrm{c}}^{k}(2), \\
-\psi_{\mathrm{L}(\mathrm{R})}^{k}(+1)-i \psi_{\mathrm{c}}^{k}(2)=(E+V) \psi_{\mathrm{c}}^{k}(3) .
\end{gathered}
$$

For the left input, the wave functions are set as $\psi_{\mathrm{L}}^{k}(-1)=$ $e^{-i k}\left(e^{-i k}+r_{\mathrm{L}} e^{i k}\right), \quad \psi_{\mathrm{c}}^{k}(1)=e^{-i k}\left(1+r_{\mathrm{L}}\right), \quad \psi_{\mathrm{c}}^{k}(3)=e^{-i k} t_{\mathrm{L}}$, and $\psi_{\mathrm{L}}^{k}(+1)=t_{\mathrm{L}}$. For the right input, the wave functions are set as $\psi_{\mathrm{R}}^{k}(-1)=t_{\mathrm{R}}, \psi_{\mathrm{c}}^{k}(1)=e^{-i k} t_{\mathrm{R}}, \psi_{\mathrm{c}}^{k}(3)=e^{-i k}\left(1+r_{\mathrm{R}}\right)$, and $\psi_{\mathrm{R}}^{k}(+1)=e^{-i k}\left(e^{-i k}+r_{\mathrm{R}} e^{i k}\right)$. After simplification, we obtain the reflection and transmission,

$$
\begin{gathered}
t_{\mathrm{L}}=t_{\mathrm{R}}=\frac{i \sin k}{\left(e^{-2 i k}-V^{2}\right) \cos k+e^{-i k}}, \\
r_{\mathrm{L}}=-\frac{\left(e^{i k}+V\right)\left(e^{-i k}-V\right)+1}{\left(e^{-2 i k}-V^{2}\right) \cos k+e^{-i k}} \cos k, \\
r_{\mathrm{R}}=-\frac{\left(e^{i k}-V\right)\left(e^{-i k}+V\right)+1}{\left(e^{-2 i k}-V^{2}\right) \cos k+e^{-i k}} \cos k .
\end{gathered}
$$

Thus, $t_{\mathrm{L}}=t_{\mathrm{R}},\left|r_{\mathrm{L}}\right|=\left|r_{\mathrm{R}}\right|$, and $\left|r_{\mathrm{L}(\mathrm{R})}\right|^{2}+\left|t_{\mathrm{L}(\mathrm{R})}\right|^{2}=1$. The scattering matrix is unitary, i.e., $S S^{\dagger}=I$, and the scattering dynamics is Hermitian-like.

In Fig. 3, we plot the reciprocal reflection probability ( $R=R_{\mathrm{L}}=R_{\mathrm{R}}$ ) and the reciprocal transmission probability ( $T=T_{\mathrm{L}}=T_{\mathrm{R}}$ ). In Fig. 3(a), $R$ and $T$ are both maximal at $k=\pi / 2$, where the input wave has the largest group velocity. The reflection and transmission probabilities monotonously
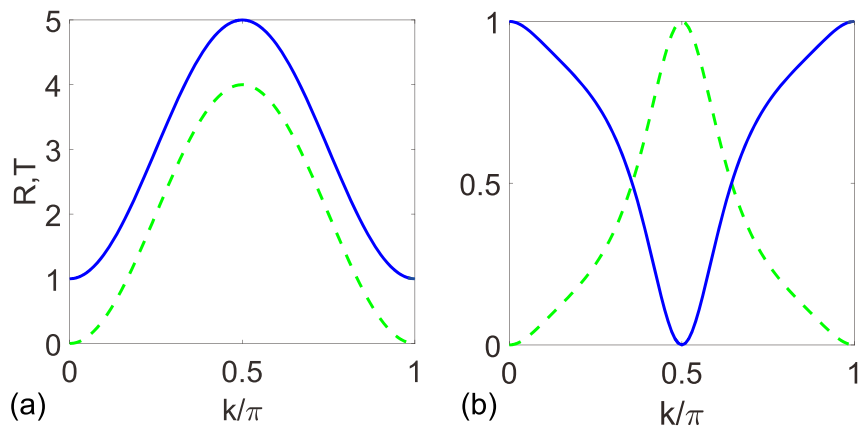

FIG. 3. The reflection (solid blue curve) and transmission (dashed green curve) probabilities as a function of the input wave vector $k$. Plots are for (a) $H_{\mathrm{c}}^{2 \mathrm{~d}}$ and (b) $H_{\mathrm{c}}^{3 \mathrm{~d}}$ at $V=1$.

increase as $V$ decreases. Both $R$ and $T$ diverge at $k=\pi / 2$ when $V=0$, and it corresponds to a spectral singularity and induces symmetric lasing toward both leads [42]. As $V$ increases, the variations on $R$ and $T$ tend to be flat. In Fig. 3(b), the reflection is zero and the transmission is unity at $k=\pi / 2$, which corresponds to a resonant transmission that is independent of on-site potentials $V$. As $V$ increases, the variations on $R$ and $T$ around $k=\pi / 2$ become sharp. Notably, the spectral singularity cannot exist in the discussed $\mathcal{P} \mathcal{T}$-antisymmetric scattering center with site number that is odd, where the scattering exhibits Hermitian behavior.

\section{SUMMARY AND DISCUSSION}

We have investigated the scattering behavior of a $\mathcal{P} \mathcal{T}$ antisymmetric non-Hermitian scattering center $H_{\mathrm{c}}$ with imaginary nearest-neighbor couplings and real on-site potentials. This type of scattering center has the feature that $\left( \pm i H_{\mathrm{c}}\right)$ satisfies the $\mathcal{P} \mathcal{T}$ symmetry. We find that the reflection $(R)$ and transmission $(T)$ are both reciprocal; and the probabilities satisfy $R \pm T=1$, which depends on the scattering center size. The scattering matrix of an even-site scattering center satisfies $\left(S \sigma_{\mathrm{z}}\right)\left(S \sigma_{\mathrm{z}}\right)^{*}=I$; an odd-site scattering center exhibits Hermitian scattering dynamics, its scattering matrix is unitary $S S^{\dagger}=I$, and no spectral singularity exists. We would like to state that all the conclusions are still valid for the scattering center with long-range imaginary couplings if all the couplings are between sites with different parity, i.e., only couplings between sites $|\mathrm{Odd}\rangle_{\mathrm{c}}$ and $\mid$ Even $\rangle_{\mathrm{c}}$ are nonzero; otherwise, only $t_{\mathrm{L}}=t_{\mathrm{R}}$ is valid because of the transpose invariant of the scattering center. Our results are useful in predicting the propagation features of $\mathcal{P} \mathcal{T}$-antisymmetric systems and their applications in optics.

\section{ACKNOWLEDGMENTS}

We acknowledge support from NSFC (Grant No. 11605094) and the Tianjin Natural Science Foundation (Grant No. 16JCYBJC40800).
[1] C. M. Bender and S. Boettcher, Phys. Rev. Lett. 80, 5243 (1998).
[2] P. Dorey, C. Dunning, and R. Tateo, J. Phys. A: Math. Gen. 34, L391 (2001). 
[3] A. Mostafazadeh, J. Math. Phys. 43, 3944 (2002).

[4] W. D. Heiss, J. Phys. A: Math. Gen. 37, 2455 (2004).

[5] H. F. Jones, J. Phys. A: Math. Gen. 38, 1741 (2005).

[6] M. Znojil, J. Phys. A: Math. Theor. 41, 292002 (2008).

[7] K. G. Makris, R. El-Ganainy, D. N. Christodoulides, and Z. H. Musslimani, Phys. Rev. Lett. 100, 103904 (2008).

[8] S. Klaiman, U. Günther, and N. Moiseyev, Phys. Rev. Lett. 101, 080402 (2008).

[9] O. Bendix, R. Fleischmann, T. Kottos, and B. Shapiro, Phys. Rev. Lett. 103, 030402 (2009).

[10] L. Jin and Z. Song, Phys. Rev. A 80, 052107 (2009).

[11] S. Longhi, Phys. Rev. A 82, 031801(R) (2010).

[12] Y. N. Joglekar, D. Scott, M. Babbey, and A. Saxena, Phys. Rev. A 82, 030103(R) (2010).

[13] A. Guo, G. J. Salamo, D. Duchesne, R. Morandotti, M. VolatierRavat, V. Aimez, G. A. Siviloglou, and D. N. Christodoulides, Phys. Rev. Lett. 103, 093902 (2009).

[14] C. E. Rüter, K. G. Makris, R. El-Ganainy, D. N. Christodoulides, M. Segev, and D. Kip, Nat. Phys. 6, 192 (2010).

[15] B. Peng, S. K. Özdemir, F. Lei, F. Monifi, M. Gianfreda, G. L. Long, S. Fan, F. Nori, C. M. Bender, and L. Yang, Nat. Phys. 10, 394 (2014).

[16] L. Feng, Z. J. Wong, R.-M. Ma, Y. Wang, and X. Zhang, Science 346, 972 (2014).

[17] H. Hodaei, M.-A. Miri, M. Heinrich, D. N. Christodoulides, and M. Khajavikhan, Science 346, 975 (2014).

[18] B. Peng, S. K. Özdemira, M. Liertzer, W. Chen, J. Kramer, H. Yilmaz, J. Wiersig, S. Rotter, and L. Yang, Proc. Nat. Acad. Sci. USA 113, 6845 (2016).

[19] H. Hodaei, A. U. Hassan, S. Wittek, H. Garcia-Gracia, R. El-Ganainy, D. N. Christodoulides, and M. Khajavikhan, Nature (London) 548, 187 (2017).

[20] W. Chen, S. K. Özdemir, G. Zhao, J. Wiersig, and L. Yang, Nature (London) 548, 192 (2017).

[21] F. Cannata, J.-P. Dedonder, and A. Ventura, Ann. Phys. (NY) 322, 397 (2007).

[22] S. Kalish, Z. Lin, and T. Kottos, Phys. Rev. A 85, 055802 (2012).
[23] Z. Ahmed, Phys. Lett. A 377, 957 (2013).

[24] A. Mostafazadeh, J. Phys. A: Math. Theor. 47, 505303 (2014).

[25] B. Zhu, R. Lü, and S. Chen, Phys. Rev. A 91, 042131 (2015).

[26] X. Q. Li, X. Z. Zhang, G. Zhang, and Z. Song, Phys. Rev. A 91, 032101 (2015).

[27] L. Jin, X. Z. Zhang, G. Zhang, and Z. Song, Sci. Rep. 6, 20976 (2016).

[28] Y. D. Chong, Li Ge, Hui Cao and A. D. Stone, Phys. Rev. Lett. 105, 053901 (2010).

[29] W. Wan, Y. Chong, L. Ge, H. Noh, A. D. Stone, and H. Cao, Science 331, 889 (2011).

[30] Y. Sun, W. Tan, H.-Q. Li, J. Li, and H. Chen, Phys. Rev. Lett. 112, 143903 (2014).

[31] D. G. Baranov, A. Krasnok, T. Shegai, A. Alù, and Y. Chong, Nat. Rev. Mater. 2, 17064 (2017).

[32] Z. Lin, H. Ramezani, T. Eichelkraut, T. Kottos, H. Cao, and D. N. Christodoulides, Phys. Rev. Lett. 106, 213901 (2011).

[33] L. Feng, Y. L. Xu, W. S. Fegadolli, M. H. Lu, J. E. B. Oliveira, V. R. Almeida, Y. F. Chen, and A. Scherer, Nat. Mater. 12, 108 (2013).

[34] R. Fleury, D. Sounas, and A. Alù, Nat. Commun. 6, 5905 (2015).

[35] H. Ramezani, H. K. Li, Y. Wang, and X. Zhang, Phys. Rev. Lett. 113, 263905 (2014).

[36] D. A. Antonosyan, A. S. Solntsev, and A. A. Sukhorukov, Opt. Lett. 40, 4575 (2015).

[37] P. Peng, W. Cao, C. Shen, W. Qu, J. Wen, L. Jiang, and Y. Xiao, Nat. Phys. 12, 1139 (2016).

[38] L. Ge and H. E. Türeci, Phys. Rev. A 88, 053810 (2013).

[39] J.-H. Wu, M. Artoni, and G. C. La Rocca, Phys. Rev. Lett. 113, 123004 (2014).

[40] J.-H. Wu, M. Artoni, and G. C. La Rocca, Phys. Rev. A 91, 033811 (2015).

[41] V. V. Konotop and D. A. Zezyulin, Phys. Rev. Lett. 120, 123902 (2018).

[42] A. Mostafazadeh, Phys. Rev. Lett. 102, 220402 (2009). 